\title{
Qualitätsmanagement und Fehlerkultur: Wie geht man mit Fehlern um?
}

Jens Roth

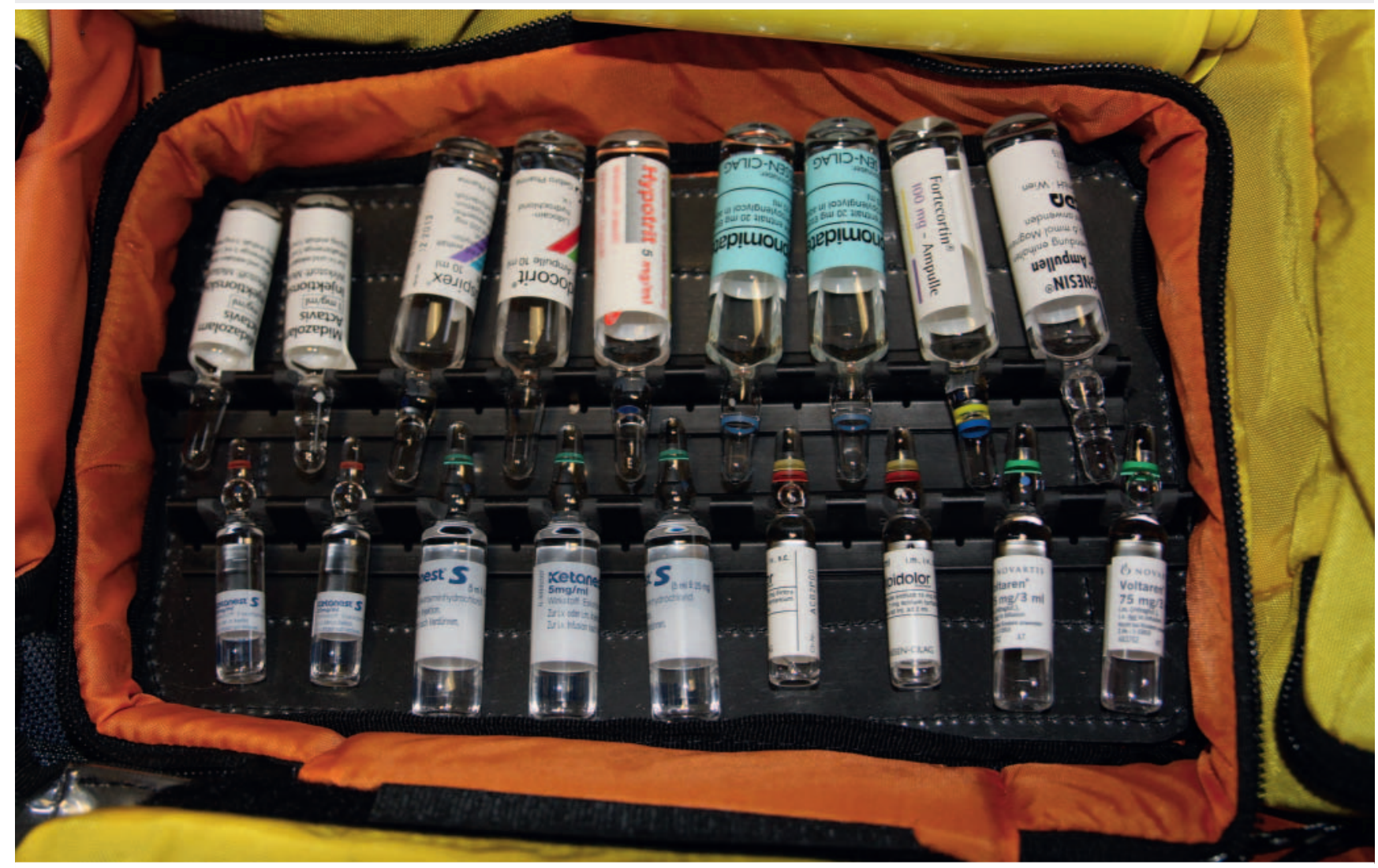

Quelle: Daniela Erhard/Thieme Gruppe

Im Rettungsdienst-Alltag mit seinen oft hektischen Situationen sind Fehler an der Tagesordnung. Eine offene Fehlerkultur und die gezielte Aufarbeitung im Rahmen eines QM-Systems können künftige Fehler vermeiden. Hier werden anhand eines Praxisbeispiels Lösungsansätze gezeigt, die sich gut in ein bestehendes QM-System integrieren lassen.

\section{DER FALL}

Ein Rettungsdienstmitarbeiter ergänzt nach einem Einsatz das verbrauchte Material in seinem Fahrzeug auf der Rettungswache. Statt des eigentlich im Einsatz benötigten Antiarrhythmikums fehlt im Ampullarium des Fahrzeugs ein Sedativum. Bei näherem Betrachten fällt ihm auf, dass beide Ampullen die gleiche Farbgebung haben und sich nur in einer sehr kleinen Schrift unterscheiden. Das Fehlen des Sedativums würde auch die unerwartete Bewusstseinsveränderung des Patienten nach der Medikamentengabe erklären. Der Patient wurde aber stabil ins Krankenhaus gebracht.
Der Rettungsdienstmitarbeiter hat 3 Möglichkeiten, mit diesem Fehler umzugehen:

1. Den Fehler für sich behalten. Angst vor Bestrafung könnte dieses Verhalten fördern. Zu erwarten wären arbeitsrechtliche Konsequenzen bis hin zu einer möglichen Kündigung. Der Fehler bleibt aber als Risiko für den nächsten Einsatz weiterhin bestehen, da die Fehlerursache nicht angegangen wurde.

2. Den Fehler sofort öffentlich machen, Notarzt und Krankenhaus verständigen. Aus fachlicher und moralischer Sicht eigentlich der erwartete Weg, jedoch bleiben die harten Konsequenzen aus dem ersten Weg.

3. Die Rettungsdienstorganisation erwartet das Auftreten von Fehlern und hat dafür Vorkehrungen getrof- 
fen. Diese Elemente sind verpflichtender Bestandteil eines funktionierenden Qualitätsmanagements (QM). Der Umgang mit Fehlern und Risiken muss unabhängig vom verwendeten QM-System geregelt sein.

\section{Fehlermanagement und Fehlerkultur}

Das reine Fehlermanagement, also die Behebung und Vermeidung von Fehlern, ist noch nicht das Gleiche wie eine Fehlerkultur. Diese Worte sollten deshalb nicht synonym verwendet werden.

Merke

Fehlerkultur ist ein ganzheitlicher Prozess.

Fehlerkultur ist deutlich vielschichtiger. Man unterscheidet drei Ebenen der Fehlerkultur [1].

\section{Ebene 1: Normen und Werte}

Darunter versteht man, wie in der Organisation mit Fehlern umgegangen wird. Dies wird maßgeblich durch die Führung beeinflusst.

Bezogen auf unseren Fall bedeutet dies: Wenn in der Wache die Suche nach dem Schuldigen und seine Bestrafung als Fehlerumgang gelebt werden, dann werden die Mitarbeiter eher Fehler vertuschen, statt sie offenzulegen. Gleiches gilt, wenn die Leitung einen offenen und transparenten Umgang bei eigenen Fehlern nicht gestattet. Werden Fehler im Wachablauf und der Organisation als belanglos abgetan, wird auch der Mitarbeiter seine eigenen Fehler so bewerten.

Die Entwicklung eines guten, offenen Verständnisses für Fehler und deren Vermeidung ist ein langwieriger Prozess. Das reine Festlegen eines Verfahrens in einem QM-Handbuch und die Benennung des Ziels „Wir wollen unsere Patienten gut und fehlerfrei versorgen " in einem Leitbild werden diese Offenheit und Akzeptanz gegenüber Fehlern nicht schaffen.

\section{Ebene 2: Kompetenzen}

In unserem Fall hat der Mitarbeiter schon eine hohe Kompetenz bewiesen, da er mit der Analyse des Fehlers begonnen hat. Er hat die vermutlich falsche Medikamentengabe erkannt, die Reaktion des Patienten als Folge daraus ermittelt und die Farbgebung der Ampulle mit der kleinen Schrift als Ursache der Verwechslung vermutet. Genau diese Kompetenzen müssen bei Mitarbeitern geschaffen und geschärft werden. Wird ein Fehler nicht erkannt, kann er immer wieder auftreten.

Fehler bestimmen unser Leben. Wir könnten nicht auf zwei Beinen laufen, wenn wir nicht vorher beim Versuch mehrfach gestürzt wären. Problematisch ist, dass unser
Hirn darauf angelegt ist, Sinn zu stiften und zu erkennen. So können Sie das Wort „Rtetnugsdeisnt“ vermutlich schnell lesen, obwohl es völlig falsch geschrieben ist.

Fehler sind keine Schande; sie helfen, Dinge zu lernen oder zu verbessern. Diese Erkenntnis muss den Mitarbeitern bekannt sein. Die entsprechenden Kompetenzen müssen wie Fachwissen regelmäßig vertieft und erweitert werden.

\section{Ebene 3: Instrumentarien}

Dies ist die umfangreichste Ebene. Im Folgenden werden gängige Instrumente der Fehleranalyse und der Fehlervermeidung vorgestellt.

\section{Instrumente der Fehleranalyse und -vermeidung}

\section{Anonymität}

Bereits 1985 entdeckte Boeing in einer Studie, dass bei fortschreitender Entwicklung des Luftverkehrs und gleichbleibender Sicherheit im Jahr 2000 jede Woche mit einem medienwirksamen Unfall zu rechnen ist. Dass das nicht eingetreten ist, liegt am verbesserten Fehlermanagement der Airlines. Unter anderem wurden dort CIRS (Critical Incident Reporting System) installiert.

Diese Systeme wurden mittlerweile auch von Rettungsdiensten in einigen Bundesländern oder von einzelnen Rettungsorganisationen eingeführt. So könnte der Mitarbeiter aus unserem Beispiel anonym in einem Meldeportal den Fehler der Medikamentenverwechslung eingeben. Die Empfänger der Nachricht bewerten die Meldung und sprechen eine Handlungsempfehlung aus - im oben genannten Fall z. B. die Beschaffung eines anderen Medikaments mit gleichem Wirkstoff, damit die optische Verwechslung ausgeschlossen ist.

Merke

Kommunikation zu Fehlern ist wichtig!

Hauptbestandteil eines jeden CIRS ist aber die Verbreitung der Meldung. Durch die Information der Mitarbeiter zu einem Vorfall werden die Sinne geschärft und es wird ein Bewusstsein für Fehler geschaffen.

PRAXIS/MAßNAHMEN

Im Rettungsdienst sollten softwarebasierte Kommunikationssysteme mit Ablieferungsnachweis eingeführt werden. So kann im Zweifel nachvollzogen werden, ob alle Mitarbeiter die Information auch gelesen haben. 
- Tab. 1 Ishikawa-Diagramm zu Medikamentenverwechslung mit jeweils einem Beispiel pro Zeile.

\begin{tabular}{|c|c|c|}
\hline Element & Ursache & Mögliche Verhinderungsmaßnahme \\
\hline Mensch/Mitarbeiter & $\begin{array}{l}\text { unsichere Auswahl des Medika- } \\
\text { ments anhand Etikettenfarbe }\end{array}$ & $\begin{array}{l}\text { Double-Check/Cross-Check durch Kollegen bei } \\
\text { Entnahme einführen }\end{array}$ \\
\hline $\begin{array}{l}\text { Material/Ausrüstung/ } \\
\text { Arbeitsumgebung }\end{array}$ & viele farbgleiche Ampullen & $\begin{array}{l}\text { bei Beschaffung auf möglichst verwechslungsfreie } \\
\text { Kennzeichnung achten }\end{array}$ \\
\hline Messung/Überwachung & $\begin{array}{l}\text { keine Gegenkontrolle durch z. B. } \\
\text { Kollegen/Arzt vor Gabe }\end{array}$ & 6R-Regel zu Medikamenten nochmals schulen \\
\hline Methode und Prozesse & $\begin{array}{l}\text { schnelles Aufziehen von Medika- } \\
\text { menten ohne definierte Kontroll- } \\
\text { punkte }\end{array}$ & $\begin{array}{l}\text { Einführung der Kontrollpunkte, z. B.: Nach Ab- } \\
\text { schluss des Aufziehens wird der Medikamenten- } \\
\text { name nochmals laut von der Ampulle vorgelesen }\end{array}$ \\
\hline Management/Führung & $\begin{array}{l}\text { keine anonyme Fehlermeldung } \\
\text { möglich }\end{array}$ & Schaffung eines Meldeportals \\
\hline Umwelt, externe Faktoren & $\begin{array}{l}\text { nachts auf der Straße keine gute } \\
\text { Sichtbarkeit im Ampullarium }\end{array}$ & $\begin{array}{l}\text { Beschaffung von kleiner Zusatzleuchte im mobilen } \\
\text { Ampullarium }\end{array}$ \\
\hline
\end{tabular}

Eine Vernetzung der vorhandenen CIRS wäre sinnvoll, ist aber bisher nicht angedacht. Dies bedeutet, dass nach der Meldung eines Vorfalls in einem CIRS derselbe Fehler noch 15-mal in anderen Bundesländern passieren müsste, bis er überall bekannt wäre. Dies widerspricht dem Ansatz risikobasierter QM-Systeme wie der gängigen DIN ISO 9001:2015. Fehler sollen möglichst nur einmal auftreten und dann offensiv angegangen werden.

\section{Merke}

Der Fehler des einen ist das Risiko des anderen und muss nachhaltig verhindert werden.

\section{Richtiges definieren}

Um einen Fehler zu erkennen, muss klar sein, wie das korrekte Handeln aussieht. Dies kann z. B. über Fallvorstellungen erreicht werden. Ein konkreter Einsatz wird im Kollegenkreis besprochen und mit der Dokumentation anhand von Leitlinien auf Richtigkeit geprüft. Im kollegialen Dialog können so Handlungsweisen besprochen und es soll der richtige Weg definiert werden. Im vorliegenden Fall wäre auch dies eine Möglichkeit, den Fehler zu besprechen.

Die Fallbesprechung kann auch anonymisiert erfolgen. Nötig ist dafür ein festes Verfahren zur Fallvorstellung, z. B. anhand eines Fragebogens und der Einsatzdokumentation. Die Vorstellung kann dann ein Kollege vornehmen, der nicht Teil des Einsatzteams war. So kann der Einsatz aufgearbeitet und der Fehler zukünftig vermieden werden, ohne dass die Beteiligten Sanktionen befürchten müssen.

Durch ein QM-System wird die Regelmäßigkeit eines solchen Vorgehens sichergestellt. Es sollte im Schulungsplan fest verankert sein. Wichtig ist auch, Einsätze zu besprechen, die nach Einschätzung des Rettungsdienstteams keine Fehler enthalten haben. Überraschenderweise wird man viele Dinge aufdecken, die verbesserungswürdig sind. Dies erfüllt einen der Grundsätze der QM-Arbeit: die kontinuierliche Verbesserung des Handelns.

\section{Gezielte Fehleranalyse}

Zur Fehleranalyse stehen viele Analysetools zur Verfügung. Eines soll hier exemplarisch vorgestellt werden: Das Ishikawa- oder Fischgrät-Diagramm ändert die Sichtweise auf ein Problem und ermöglich dadurch eine umfassende, strukturierte Aufarbeitung des Fehlers. Wichtige Elemente sind die Ursachen Mensch, Material, Messung, Methode, Management und Umwelt. Unser Beispielfall könnte wie in $>$ Tab. 1 analysiert werden.

Die Analyse sollte immer anlassbezogen stattfinden. Sinnvoll ist dies im Abteilungs- oder Wachenteam. Bei großen Wachen kann auch ein repräsentativ zusammengestelltes Qualitätsteam die Analyse durchführen. Leitung, Mitarbeiter und QM-Verantwortliche sollten Bestandteil sein. Nach der Analyse ( $\triangleright$ Abb. 1) kann sofort ein Verbesserungsvorschlag eingebracht werden.

\section{PRAXIS/MABNAHMEN}

Da hier offen Probleme auch im Leitungsbereich angesprochen werden sollen, könnte eine externe Begleitung (z. B. durch eine andere Abteilung im Unternehmen) als neutraler Dritter sinnvoll sein.

Nachhaltig sind die Maßnahmen nur durch eine Wirksamkeitsprüfung: Für alle Maßnahmen sollte nach einem gewissen Zeitabstand hinterfragt werden, ob das angestrebte Ziel auch erreicht wurde. Ein QM-System wird diese Aufgabe automatisch leisten, da in ihm immer Maßnahmen nachverfolgt und überwacht werden müssen. 


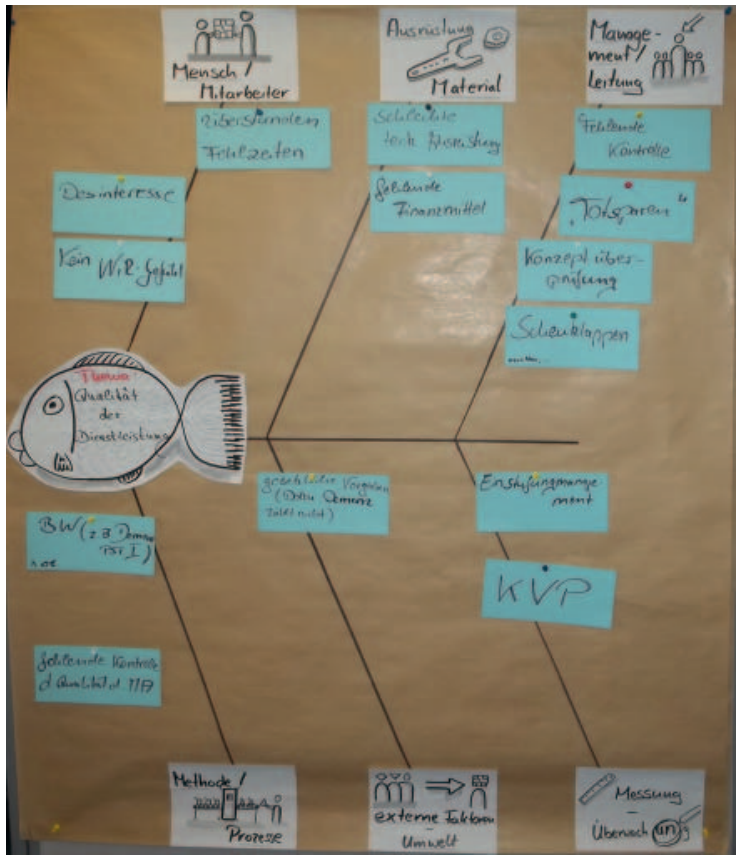

- Abb. 1 Umsetzung des Ishikawa-Diagramms in einer Teamsitzung.

\section{Resümee}

Ein Rettungsdienst, der nachhaltig versucht, eine Fehlerkultur zu etablieren, wird auf lange Sicht deutlich weniger Fehler machen. Dies verbessert alle Bereiche des Unternehmens, vor allem die Patientenversorgung - und das sollte ja das oberste Ziel eines Rettungsdienstes sein.

Leider ist dieser Aspekt bei QM-Systemen meist unterrepräsentiert. QM-Systeme sind auf eine Verbesserung der Prozesse angelegt. Die Dienstleistung ist eher zweitrangig.

Eine Fehlerkultur und der gezielte Umgang mit Fehlern helfen, dieses Manko auszugleichen. Insgesamt wird sich auch das Bewusstsein des Unternehmens mit seinen Mitarbeitern positiv entwickeln, da alle in die Prozesse der Fehlerkultur eingebunden sind.

\section{KERNAUSSAGEN}

- Fehlerkultur ist ein unverzichtbarer Baustein, um in einem fehleranfälligen Bereich wie einem Rettungsdienst - auch im Interesse der Mitarbeiter eine gute Dienstleistung erbringen zu können.

- Die Einführung einer Fehlerkultur mit Unterstützung durch ein bestehendes QM-System ist ein langfristiger Prozess. Sie kann nicht durch einfache Verfahrensanweisungen spontan umgesetzt werden.
- Alle Bereiche des Unternehmens müssen integriert werden, von der Führung bis zum kleinsten Mitarbeiter, damit die Aufgabe gelingen kann.

- Die Schuldfrage beim Auftreten eines Fehlers ist nachrangig. Wichtiger ist die Frage: Warum ist ein Fehler aufgetreten?

- Fehlerkultur braucht motivierte, kompetente Mitarbeiter, die im Rahmen von QM-Vorgaben gezielt die Instrumente der Fehleranalyse anwenden.

\section{Interessenkonflikt}

Die Autoren geben an, dass kein Interessenkonflikt besteht.

\section{Autorinnen/Autoren}

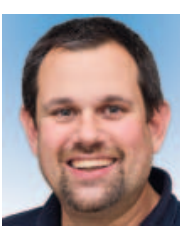

\section{Jens Roth}

ist bayernweit für die Qualitätsarbeit und den Datenschutz beim ASB verantwortlich, Lehrkraft an den ASB-Schulen Bayern für Notfallsanitäter und Praxisanleiter und Fachwirt im Gesundheits- und Sozialwesen.

Korrespondenzadresse

Jens Roth

ASB Schulen Bayern gGmbH

Eichenhainstraße 30

91207 Lauf/Pegnitz

roth@asb-schulen.de

\section{Literatur}

[1] Schüttelkopf EM. Fehlerkultur. Zu Begriff, Bedeutung und Bewertung der organisationalen Fehlerkultur. Wien: PeterLang-Verlag; 2006

\section{Bibliografie}

DOI https://doi.org/10.1055/a-0495-1653

retten 2018; 7: 322-325

(c) Georg Thieme Verlag KG, Stuttgart · New York ISSN 2193-2387 